\title{
ROOT-ENDOPHYTIC FUNGI DIVERSITY OF FUZI (ACONITUM CARMICHAELII) AND THEIR ANTI-FUNGAL ACTIVITY
}

\author{
LV, Y. ${ }^{1,2,3 \#}-$ SHEN, Y. ${ }^{5 \#}-$ SHI, Y. ${ }^{6}-$ SHEN, F. ${ }^{4}-$ CHEN, Y. ${ }^{4}-$ ZI, S. ${ }^{5}-$ ZHU, Z. ${ }^{2 *}-$ KenNELLY, E. J. ${ }^{7,8^{*}}-$ \\ ZHAO, D. ${ }^{1,2,3^{*} \#}$ \\ ${ }^{1}$ Laboratory of Ecology and Evolutionary Biology, State Key Laboratory for Conservation and \\ Utilization of Bio-Resources in Yunnan University, Kunming 650091, China \\ ${ }^{2}$ Yunnan Institute of Materia Medica, Yunnan Baiyao Group Company Limited, Kunming \\ 650111, China \\ ${ }^{3}$ Key Laboratory of Tropical Plant Resources and Sustainable Use, Xishuangbanna Tropical \\ Botanical Garden, Chinese Academy of Sciences, Kunming 650000, China \\ ${ }^{4}$ School of Agriculture, Yunnan University, Kunming 650504, China \\ ${ }^{5}$ College of Agriculture and Biotechnology, Yunnan Agricultural University, Kunming, China \\ ${ }^{6}$ Institute of Medicine Plants, Yunnan Academy of Agricultural Sciences, Kunming 650201, \\ People's Republic of China \\ ${ }^{7}$ Department of Biological Sciences, Lehman College, City University of New York, New York \\ 10468, United States of America \\ ${ }^{8}$ Ph.D. Programs in Biochemistry, Biology, and Chemistry, The Graduate Center, City \\ University of New York, New York 10468, United States of America \\ ${ }^{\#}$ These authors contributed equally to this work. \\ *Corresponding authors \\ e-mail: zzy1370@163.com (Z.Zhu),Edward.kennelly@lehman.cuny.edu (E.J. Kennelly), \\ zhaodk2012@ynu.edu.cn(D.Zhao) \\ (Received $8^{\text {th }}$ Jul 2019; accepted $16^{\text {th }}$ Oct 2019)
}

\begin{abstract}
Endophytic fungi (EFs) are one of the important and quantifiable components of fungal biodiversity. In the study, 171 endophytic fungi were isolated from the roots of Aconitum carmichaelii, a traditional Chinese medicine, collected from six production sites of Yunnan Province in southwestern China. These EFs were categorized to belong to 28 taxa by molecular identification, and Chaetomium and Fusarium were the dominant genera with a relative frequency of $16.96 \%$ and $16.37 \%$ respectively. Cladosporium and Pochonia were found to be unique dominant genera from the roots of A. carmichaelii in Huize and Yulong counties, respectively. The Shannon index ranged from 1.68 to 2.05 and the Simpson's diversity index ranged from 0.81 to 0.92 for the EFs of $A$. carmichaeli, indicating the rich diversity of EFs in A. carmichaelii. Preliminary screening of the growth inhibition of three severe fungal pathogens by the isolated EFs was conducted and 17 genera of EFs were found to inhibit the growth of the tested pathogens. For example, Acrostalagmus luteoalbus, Phoma sp., and Thielavia sp. each displayed good anti-fungal activity against Alternaria alternate, Fusarium oxysporum f. sp. cucumerinum, and Phytophthora drechleri. The investigation of the root-EFs diversity of A. carmichaelii provides additional fungal resources with the potential to biologically control pathogenic fungi.

Keywords: traditional Chinese medicine, fungal biodiversity, community composition, ITS sequence, antagonism against fungal pathogens
\end{abstract}




\section{Introduction}

Endophytic fungi (EFs), well-studied to be potential resources for producing bioactive natural products (Aly et al., 2008), asymptomatically infect healthy plant tissues for all, or at least a significant part of their life cycles (Hyde and Soytong, 2008). The diversity of endophytes has been fully investigated in the case of many plant taxa (Sun et al., 2011; Silva et al., 2012; Kohout et al., 2013; Fernandes et al., 2015; Nascimento et al., 2015), and endophytes were shown to increase ecological adaption by protecting plants against biotic and abiotic stresses (Liu et al., 2009; Morsy et al., 2010; Bittleston et al., 2011).

Nowadays, drug-resistance among bacteria and fungi is spreading significantly, and therefore further search is urgently needed for new and effective antimicrobial agents. Endophytes have the potential to produce bioactive compounds, such as alkaloids, resulting in the improvement of the evolutionary fitness of their host by providing resistance against micro-pathogens (Strobel et al., 2004; Kusari and Spiteller, 2012; Newman, 2015). EFs can produce the antimicrobial compounds with inhibitory activity toward pathogenic fungi (Li et al., 2005), and therefore can be helpful in fungal biocontrol in agricultural production (Mishra et al., 2012).

Aconitum L. is a large genus of more than 400 species, mainly distributed in the temperate regions of the northern hemisphere (Xiao et al., 2006). Aconitum carmichaelii, a perennial plant of the Ranunculaceae family, is one of the famous traditional Chinese medicines (TCM) fuzi (附子). As one of the most important TCMs, it has been used extensively by humans as a cardiotonic, to protect cardiomyocytes, as an analgesic and to treat arrhythmia (Zhou et al., 2015). However, there has been only one preliminary study about the EFs in Aconitum (Li et al., 2009). We aimed to isolate and identify naturally-occurring EFs in A. carmichaelii roots collected from the main production area of Yunnan Province, China, and explore their potential inhibitory activity against three primary fungal pathogens, Alternaria alternate, Fusarium oxysporum f. sp. cucumerinum, and Phytophthora drechsleri.

\section{Materials and methods}

\section{Plant materials}

The roots of A. carmichaelii were sampled in September 2014 across six selected counties including Heqing, Huize, Luquan, Malong, Panlong, and Yulong in Yunnan Province, southwestern China (Fig. 1), in order to maximize the endophytic fungal diversity. These A. carmichaelii were cultivated by local people. Five plants were randomly selected from each site, from which 3-5 lateral roots were collected. The roots and associated soil were packaged separately in sealable plastic bags, immediately transported to the laboratory, and then stored at $4{ }^{\circ} \mathrm{C}$ for isolating of endophytic fungi.

\section{Endophyte isolation and primary classification based on morphological traits}

The collected roots were washed under running tap water for $5 \mathrm{~min}$, sterilized in $75 \%$ ethanol for $1 \mathrm{~min}$, rinsed once in sterile water, $2 \% \mathrm{NaOCl}$ for $5 \mathrm{~min}$, and then rinsed three times in sterile water. After drying the roots on sterile paper, they were cut into segments, each about $1 \mathrm{~cm}$ long. From each of the six collections, we randomly selected 45 segments, and divided into them into three groups with 15 segments each to comprise a replicate sample. The samples were placed in Petri dish containing potato 
dextrose agar (PDA) medium amended with $0.5 \mathrm{~g} / \mathrm{L}$ streptomycin sulfate, with one segment per plate. The plates were incubated at $28^{\circ} \mathrm{C}$ and checked every other day for 3 weeks; and the fungi growing out of the roots were transferred to fresh PDA plates. Primary classification was carried out based on mycelia and colony morphological traits and secretion. After that, at least 50\% isolates in each group were randomly selected for the molecular identification.

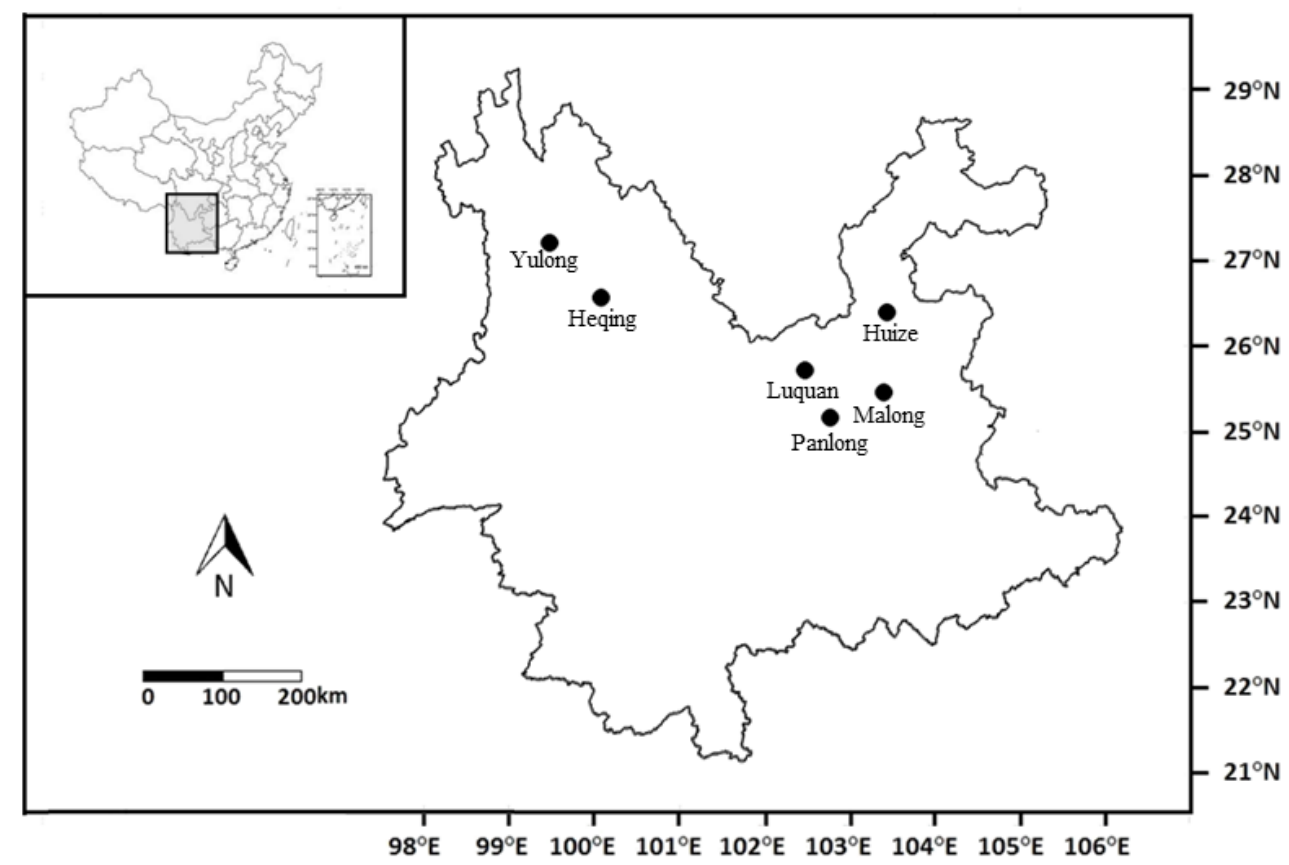

Figure 1. The geographical locations from left to right represent Yulong, Heqing, Luquan, Panlong, Malong, and Huize counties

\section{Fungal identification by DNA analysis}

Fungal DNA was extracted from pure culture mycelia using a $1 \times$ cetyltrimethylammonium bromide (CTAB) method (Niu et al., 2010). The internal transcribed spacer (ITS) region of nuclear rDNA was amplified with the universal eukaryotic primer pair ITS1 (5'-CTTGGTCATTTAGAGGAAGTAA-3') and ITS4 (5'TCCTCCGCTTATTGATATGC-3'). PCR amplifications were performed in a reaction mixture containing $2 \times$ PCR EasyTaq PCR SuperMix, DNA extracts, and the primer pair. Thermocycler settings were 5 min initial denaturation at $94{ }^{\circ} \mathrm{C}$, followed by 35 cycles of $30 \mathrm{~s}$ at $94{ }^{\circ} \mathrm{C}, 50 \mathrm{~s}$ at $53{ }^{\circ} \mathrm{C}, 1 \mathrm{~min}$ at $72{ }^{\circ} \mathrm{C}$, with a $10 \mathrm{~min}$ final extension at $72{ }^{\circ} \mathrm{C}$. Sequences were manually inspected and edited using Chromas 2.4.1. Putative identification of sequences was carried out by comparing consensus sequences to the National Center for Biotechnology Information (NCBI) DNA database using the Basic Alignment Search Tool (BLAST). The following parameters were used to determine significance of BLAST results: alignment score $>500, \mathrm{E}<0.0001$, and over $97 \%$ sequence similarity were identified as the same species. The ITS sequences of those isolates were deposited in NCBI (MF962929-MF963015). Through sequences analysis in comparison with each other, phylogenetic tree figure of the isolated EFs was constructed using neighbor-joining (NJ) methods with 1000 boot-strap replications using MEGA 6.0. 


\section{Analyses of relative frequency and diversity index}

The colonization rate was calculated as follows (Eq. 1) ( $\mathrm{Su}$ et al., 2010), where $\mathrm{N}_{\mathrm{i}}$ is the total number of segments colonized by endophytes, and $\mathrm{N}_{\mathrm{t}}$ is the total number of segments.

$$
\text { colonization rate }(\%)=\frac{N_{i}}{N_{t}} \times 100
$$

The relative frequency (RF \%) (Yuan et al., 2010) was calculated according to the following formula (Eq. 2), where $\mathrm{n}_{\mathrm{i}}$ is the number of isolates of one species divided, and the $\mathrm{N}$ is the total number of isolates.

$$
\mathrm{RF}(\%)=\frac{\mathrm{n}_{\mathrm{i}}}{\mathrm{N}} \times 100
$$

The Shannon index (H') (Shannon and Weiner, 1963) was calculated according to the following formula (Eq. 3), where $\mathrm{k}$ is the total species number of one plot and $P_{i}$ is the relative abundance of endophytic fungal species in the community.

$$
\mathrm{H}^{\prime}=-\sum_{\mathrm{i}=1}^{\mathrm{k}}\left(P_{\mathrm{i}} \times \ln P_{\mathrm{i}}\right)
$$

The fungal dominance was determined by Camargo's index (Eq. 4) (Hunter and Gaston, 1988), where $S$ was species richness = the number of species present in any given sample. A species was defined as dominant if $P_{i}>$ Camargo's index.

$$
\text { Camargo' s index }=\frac{1}{\mathrm{~S}}
$$

Simpson's index (D) and Simpson's diversity index (1-D) (Camargo, 1993), where D was calculated as (Eq. 5). D could range between 0 (infinite diversity) and 1 (no diversity).

$$
\mathrm{D}=\sum_{\mathrm{i}=1}^{\mathrm{k}}\left(\frac{\mathrm{n}_{\mathrm{i}} \times\left(\mathrm{n}_{\mathrm{i}}-1\right)}{\mathrm{N} \times(\mathrm{N}-1)}\right)
$$

\section{Anti-fungal activity assays}

The anti-fungal activity of the EFs was evaluated against three fungal pathogens: Alternaria alternate, Fusarium oxysporum f. sp. cucumerinum, and Phytophthora drechsleri (all provided by Professor Zhiwei Zhao of the State Key Laboratory for Conservation and Utilization of Bio-Resources, Yunnan University). A dual-culture technique was modified to study antagonism (Gao et al., 2002). The pathogen was inoculated on PDA medium center and four different isolated fungi were inoculated around pathogen, incubated at $28{ }^{\circ} \mathrm{C}$ for a week, and the anti-fungal zone were measured by ruler ( $\mathrm{mm})$. 


\section{Results}

\section{Colonization rates and diversity of EFs}

Colonization rate is an indication of the number of EFs in host plants and varies with the altitude, humidity, precipitation, temperature and plant community (Hashizume et al., 2010). In the present study, the colonization rate of the EFs from six sites ranged from $46.7 \%$ to $73.3 \%$, and the highest colonization rate appeared in Malong, while the lowest was appeared in Panlong (Table 1). To further investigate the biodiversity of the EFs, we calculated the Shannon diversity index (H'), Simpson's index (D) and Simpson's diversity index (1-D). The Shannon diversity indexes ( $\left.\mathrm{H}^{\prime}\right)$ of the endophytic community in the six different sites were $1.98,1.88,1.82,1.76,2.05$ and 1.68, respectively. The Simpson's diversity index is between 1 and 0 , with 1 representing infinite variety of diversity, and 0 representing no diversity. The Simpson's diversity index showed a similar pattern for the six sites, which were $0.92,0.85$, $0.88,0.89,0.88$ and 0.81 , respectively.

Table 1. Number, colonization rate, Shannon-Weiner index ( $\left.H^{\prime}\right)$ and Simpson's diversity index (1-D) of the root-endophytic fungi $(E F)$ of A. carmichaelii from the six regions of Yunnan, China

\begin{tabular}{c|c|c|c|c}
\hline Collection site & No. of EFs isolated & Colonization rate (\%) & $\mathbf{H}^{\prime}$ & 1-D \\
\hline Heqing & 26 & 48.9 & 1.98 & 0.92 \\
Huize & 44 & 60.0 & 1.88 & 0.85 \\
Luquan & 38 & 64.4 & 1.82 & 0.88 \\
Malong & 38 & 73.3 & 1.76 & 0.89 \\
Panlong & 26 & 46.7 & 2.05 & 0.88 \\
Yulong & 39 & 53.3 & 1.68 & 0.81 \\
Total & 171 & 57.8 & & \\
\hline
\end{tabular}

\section{Community composition of EFs}

A total of 171 isolates were obtained from collected roots, and identified into 28 taxa by molecular identification based on rDNA ITS sequence analysis (Table 2). A total of 10 endophytic taxa from Heqing; 12 taxa were identified from Huize; 10 taxa from Luquan; 12 taxa from Malong; 11 taxa from Panlong; and 10 taxa from Yulong (Table 2). The dominant taxa determined by the Camargo's index and $P_{i}$ were Chaetomidium arxii, Chaetomium sp., and Phoma sp. in Heqing; Chaetomium sp., Cladosporium sp., and Fusarium sp. in Huize; Chaetomidium arxii, Plectosphaerella sp., and Pochonia sp. in Luquan; Chaetomium sp. and Plectosphaerella sp. in Malong; Chaetomidium arxii, Chaetomium globosum, Ilyonectria sp., Phoma sp., and Plectosphaerella sp. in Panlong; and Fusarium oxysporum and Ilyonectria sp. in Yulong. Only Chaetomidium arxii and Plectosphaerella sp. were identified in all six sites (Table 2). The rates of colonization for Chaetomium sp. in Huize and Fusarium oxysporum in Yulong were higher than in other sites (Table 2). Endemic endogenous fungi were isolated from five habitats of $A$. carmichaelii, including: Huize (Cladosporium sp., Cylindrocarpon sp., Mucor racemosus, and Verticillium tricorpus,); Luquan (Bjerkandera sp., Pochonia sp., and Thielavia sp.); Malong (Acrostalagmus luteoalbus and Trametes versicolor); Panlong (Daldiniafissa sp.); and Yulong (Clonostachys rosea and Paraphaeosphaeria sporulosa). The phylogenetic tree indicated that Chaetomium sp., Fusarium sp. and Plectosphaerella sp. were the 
dominant EFs in this medicinal plant. In this study, phylogenetic analysis revealed that all sequences clustered in at least 18 discrete sequence groups (Fig. 2). The majority of clones (73.08\%) fell into the G 1 (Group 1), G 2 (Group 2) and G 4 (Group 4), which were distributed across most root samples.

\section{Anti-fungal activity assays}

The diversity and antimicrobial potential of EFs from a number of medicinal plants have been studied (Qin et al., 2009; Egamberdieva et al., 2017; Xiang et al., 2017). Our screening of anti-fungal activity of the isolated EFs identified 17 genera which are resistant to the three fungal pathogens (Alternaria alternate, Fusarium oxysporum $\mathrm{f}$. sp. cucumerinum, and Phytophthora drechsleri) in agricultural production (Table 3). Acrostalagmus luteoalbus (LD34), Phoma sp. (MC6), and Thielavia sp. (LQ14) displayed good anti-fungal effects, and the anti-fungal zone for Alternaria alternate, Fusarium oxysporum f. sp. cucumerinum, and Phytophthora drechsleri were 4, 3, and $2 \mathrm{~mm} ; 5,3$, and $3 \mathrm{~mm}$; and 4,3 , and $3 \mathrm{~mm}$, respectively, although they accounted for the small proportion of the colonization (Table 3). In addition, Chaetomium sp. and Fusarium sp., two dominant strains obtained in this experiment, also showed some antifungal activity (Table 3).

Table 2. Number, taxa and relative frequency $(R F)$ of the root-EFs of A. carmichaelii collected from six regions of Yunnan, China

\begin{tabular}{|c|c|c|c|c|c|c|c|}
\hline \multirow{2}{*}{ Taxa } & \multicolumn{7}{|c|}{ No. of strains isolated from each regions (RF \%) } \\
\hline & Heqing & Huize & Luquan & Malong & Panlong & Yulong & Total (RF \%) \\
\hline Acrostalagmus luteoalbus & & & & $1(2.63)$ & & & $1(0.58)$ \\
\hline Alternaria sp. & & & $1(2.63)$ & & $1(3.85)$ & & 2(1.17) \\
\hline Bjerkandera sp. & & & $1(2.63)$ & & & & $1(0.58)$ \\
\hline Chaetomidium arxii & $3(11.54)$ & $2(4.55)$ & $6(15.79)$ & $1(2.63)$ & $3(11.54)$ & $1(2.56)$ & $16(9.36)$ \\
\hline Chaetomium globosum & & $2(4.55)$ & & & $3(11.54)$ & & $5(2.92)$ \\
\hline Chaetomium sp. & $3(11.54)$ & $12(27.27)$ & & $6(15.79)$ & $1(3.85)$ & $2(5.13)$ & $24(14.04)$ \\
\hline Cladosporium sp. & & $6(13.64)$ & & & & & $6(3.51)$ \\
\hline Clonostachys rosea & & & & & & $2(5.13)$ & $2(1.17)$ \\
\hline Cryptococcus sp. & $1(3.85)$ & & $1(2.63)$ & & & & $2(1.17)$ \\
\hline Cylindrocarpon sp. & & $1(2.27)$ & & & & & $1(0.58)$ \\
\hline Daldinia fissa & & & & & $1(3.85)$ & & $1(0.58)$ \\
\hline Fusarium oxysporum & $1(3.85)$ & $2(4.55)$ & & $3(7.89)$ & & $11(28.21)$ & $17(9.94)$ \\
\hline Fusarium sp. & $2(7.69)$ & $4(9.09)$ & & $2(5.26)$ & $1(3.85)$ & $2(5.13)$ & $11(6.43)$ \\
\hline Geomyces sp. & & & & $1(2.63)$ & $1(3.85)$ & & $2(1.17)$ \\
\hline Ilyonectria sp. & $4(15.38)$ & & $2(5.26)$ & $1(2.63)$ & $1(3.85)$ & $9(23.08)$ & $17(9.94)$ \\
\hline Irpex lacteus & & & & $1(2.63)$ & & $1(2.56)$ & $2(1.17)$ \\
\hline Mortierella sp. & & $1(2.27)$ & & $1(2.63)$ & & & $2(1.17)$ \\
\hline Mucor racemosus & & $1(2.27)$ & & & & & $1(0.58)$ \\
\hline Paraphaeosphaeria sporulosa & & & & & & $1(2.56)$ & $1(0.58)$ \\
\hline Penicillium sp. & $1(3.85)$ & $1(2.27)$ & $2(5.26)$ & & & & $4(2.34)$ \\
\hline Phoma sp. & $3(11.54)$ & & & & $6(23.08)$ & $2(5.13)$ & $11(6.43)$ \\
\hline Plectosphaerella $\mathrm{sp}$. & $2(7.69)$ & $3(6.81)$ & $5(13.16)$ & $6(15.79)$ & $6(23.08)$ & $1(2.56)$ & $23(13.45)$ \\
\hline Pleosporales sp. & & & & $1(2.63)$ & $1(3.85)$ & & $2(1.17)$ \\
\hline Pochonia sp. & & & $7(18.42)$ & & & & $7(4.09)$ \\
\hline Thielavia sp. & & & $3(7.89)$ & & & & $3(1.75)$ \\
\hline Trametes versicolor & & & & $2(5.26)$ & & & $2(1.17)$ \\
\hline Trichocladium sp. & $2(7.69)$ & & $2(5.26)$ & & & & $4(2.34)$ \\
\hline Verticillium tricorpus & & $1(2.27)$ & & & & & $1(0.58)$ \\
\hline Total taxa recovered & 10 & 12 & 10 & 12 & 11 & 10 & 28 \\
\hline
\end{tabular}




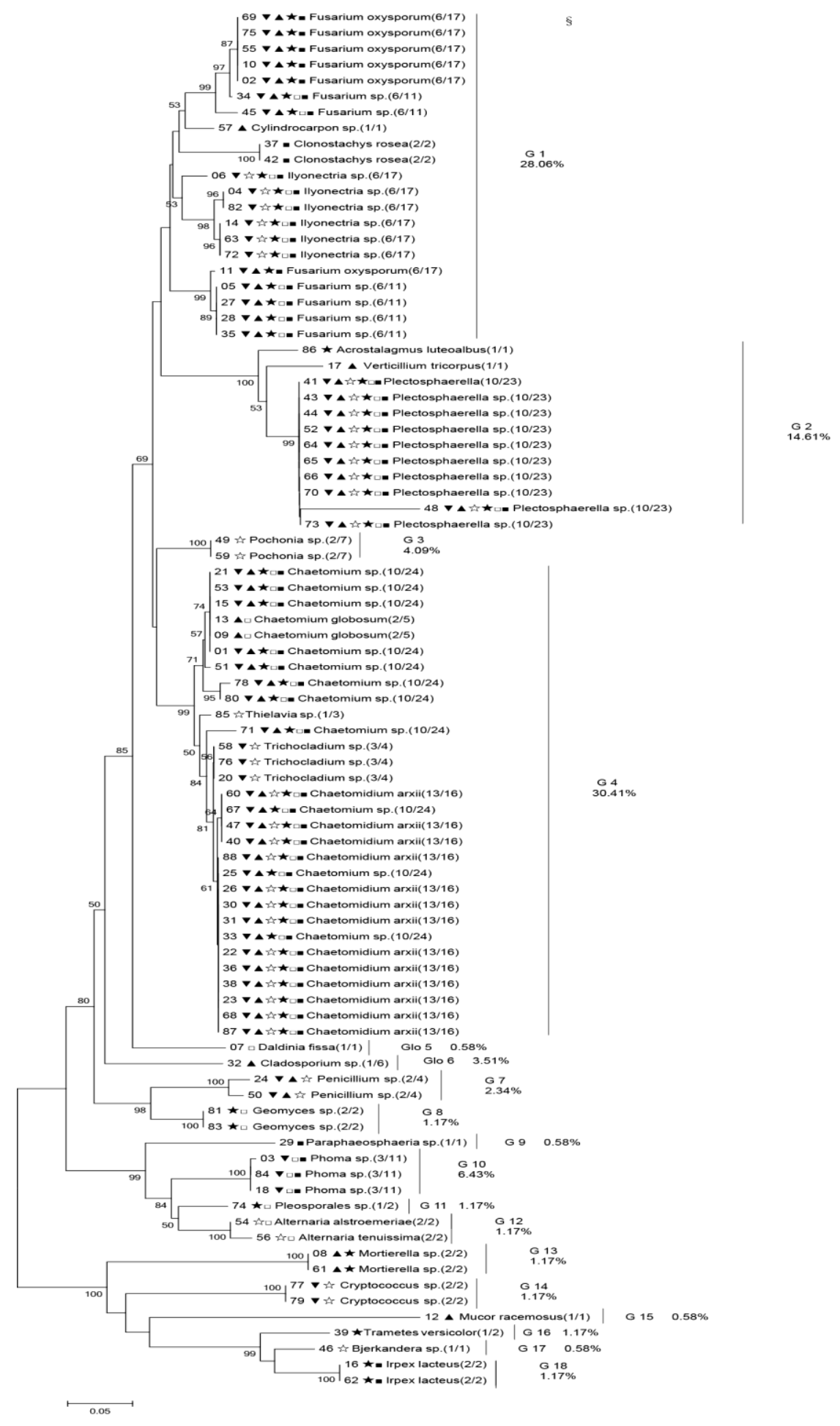

Figure 2. Neighbor-joining tree of ITS sequence of endophytic fungi associated with Aconitum carmichaelii constructed with a 1000 boot-strap replications. Bar: $0.5 \%$ sequence divergence. Numbers above the nodes indicate bootstrap support in neighbor-joining analysis. Representative sequences with Anti-black triangles ( $\mathbf{\nabla})$, black triangles $(\mathbf{\Delta})$, white pentagram $(\lesssim)$ ), black pentagram ( $\star$ ), white square ( $\square$ ), black square (ロ) represent EFs originating from Heqing, Huize, Luquan, Malong, Panlong, Yulong. Arabic numbers in parentheses indicate the number of sequences (above slash) and the number of sequenced clones (below slash). $\S$ : represents the ratio of the numbers of sequences in a given clade to the total sequenced clones 
Table 3. The anti-fungal activity of EF collected from A. carmichaelii

\begin{tabular}{|c|c|c|c|c|}
\hline \multirow[b]{2}{*}{ Taxa } & \multirow[b]{2}{*}{ No. } & \multicolumn{3}{|c|}{ Pathogen fungi (mm) } \\
\hline & & $\begin{array}{c}\text { Alternaria } \\
\text { alternate }\end{array}$ & $\begin{array}{l}\text { Fusarium oxysporum } \\
\text { f. sp. Cucumerinum }\end{array}$ & $\begin{array}{c}\text { Phytophthora } \\
\text { drechsleri }\end{array}$ \\
\hline Acrostalagmus luteoalbus & ML20 & 3 & 3 & 3 \\
\hline Alternaria sp. & PL16 & 1 & 1 & 2 \\
\hline Chaetomidium arxii & LQ30 & 1 & 1 & 2 \\
\hline Chaetomidium arxii & PL1 & 1 & 2 & 1 \\
\hline Chaetomidium arxii & PL18 & 1 & 1 & 1 \\
\hline Chaetomidium arxii & YL15 & 1 & & 2 \\
\hline Chaetomium sp. & HZ34 & 1 & 1 & 1 \\
\hline Chaetomium sp. & HZ35 & 2 & & \\
\hline Chaetomium sp. & HZ36 & 1 & 1 & 1 \\
\hline Chaetomium sp. & ML7 & 2 & & 2 \\
\hline Chaetomium sp. & ML11 & 1 & 1 & 2 \\
\hline Clonostachys rosea & YL32 & 1 & & 1 \\
\hline Cylindrocarpon sp. & HZ15 & 1 & & 1 \\
\hline Fusarium oxysporum & YL17 & & 2 & 1 \\
\hline Fusarium sp. & HQ1 & & & 2 \\
\hline Fusarium sp. & HZ11 & 1 & & 1 \\
\hline Ilyonectria sp. & ML4 & 2 & 1 & 1 \\
\hline Ilyonectria $\mathrm{sp}$. & PL13 & 2 & & 2 \\
\hline Ilyonectria sp. & YL34 & 3 & 3 & 4 \\
\hline Mortierella sp. & HZ39 & 1 & 1 & 1 \\
\hline Paraphaeosphaeria sporulosa & YL30 & 2 & & 1 \\
\hline Phoma sp. & HQ6 & 3 & 3 & 5 \\
\hline Plectosphaerella sp. & HZ43 & 1 & 1 & 1 \\
\hline Plectosphaerella sp. & PL17 & 1 & 2 & 2 \\
\hline Pleosporales sp. & PL20 & & 2 & 1 \\
\hline Thielavia sp. & LQ14 & 2 & 3 & 4 \\
\hline Trichocladium sp. & HQ24 & 1 & 1 & \\
\hline Trichocladium sp. & LQ22 & 2 & 2 & \\
\hline Verticillium tricorpus & HZ41 & & 1 & 2 \\
\hline
\end{tabular}

The values of the anti-fungal zone in the table are the average of the three experiments. HQ, HZ, LQ, ML, PL, and YL are indicative of Heqing, Huize, Luquan, Malong, Panlong, and Yulong, respectively and the number after abbreviations showed the isolation order of the endophytic fungi

\section{Discussion}

Endophytic fungi were isolated from Aconitum carmichaelii, one of the most important TCMs, which laid the foundation for future biological control research. In this study, roots of $A$. carmichaelii were collected from six sites in Yunnan Province, the People's Republic of China for the isolation, identification and antibacterial analysis of EFs. Colonization rate and Shannon diversity index (H') of the EFs in different areas (Table 1) indicated that there were rich root-fungal endophytes existing in $A$. carmichaelii in Yunnan. In all isolates, only Chaetomidium arxii and Plectosphaerella sp. were identified in all six sites (Table 2), suggesting that these two endophytic fungi 
are host-generalists. The rates of colonization for Chaetomium sp. in Huize and Fusarium oxysporum in Yulong were higher than in other sites (Table 2). This may be due to the fact that the roots are persistent, and can form unique endophytic fungal community. In addition, Huize has been documented heavy metal contamination (Lu et al., 2014), and Chaetomium sp. is known as a soil and contaminant fungi (Abdel-Lateff, 2008). Endemic EFs were isolated from five habitats, and the population structure of endophytic fungi normally represents a certain degree of regional specificity (Jia et al., 2016). In contrast to the endemic EFs, dominate endophytic fungi lived in a wide variety of plants such as Phoma sp. (Nisa et al., 2015), which was also isolated from $A$. carmichaelii with a RF as $6.43 \%$.

Screening of anti-fungal activity of the isolated EFs identified 29 isolates, such as Chaetomium sp. (Table 3), with wide-spectrum antibacterial activity (Li et al., 2009). Previous research showed that Chaetomium sp. suppressed the growth of bacteria and fungi through direct competition, mycoparasitism, and antibiosis (Rodríguez et al., 2002). Two antibacterial furano-polyenes, (-) - musanahol and 3-epi-aureonitol, were isolated from cultures of Chaetomium sp. (Marwah et al., 2007). Dutch elm disease, caused by the fungal pathogen Ceratocystis ulmi, can be controlled by the endophytic fungus Phomopsis oblonga (Webber, 1981). Leutou et al. (2016) concluded that the microbial transformation of anthranilic acid by the marine-mudflat-derived fungus Thielavia sp. produced an antibacterial polycyclic quinazoline alkaloid, thielaviazoline. Fusarium oxysporum is well represented in the rhizosphere microflora (Lecomte et al., 2016). Some isolates can induce wilt or root rots while others are considered as nonpathogenic taxa. For example, nonpathogenic $F$. oxysporum in soil can suppress Fusarium wilts (Fravel et al., 2003), and the strain has also attracted interest for soil bioremediation and water purification due to its capability to detoxify and colonize polluted environments (Steinberg et al., 2016). Ratnaweera et al. (2015) found that equisetin, isolated from endophytic Fusarium sp., exhibited antibacterial activities against the Gram-positive bacteria.

Some recent studies have shown that Lentzea sp., Nigrospora sp. and Streptomyces sp. isolated from A. carmichaelii have antibiotic activity (Qiu et al., 2015; Zhang et al., 2016). EFs isolated from medicinal plants sometimes produce similar compounds as their hosts (Newman, 2015). Diterpene alkaloids are the dominant secondary metabolites of Aconitum species (Zhou et al., 2015), and other diterpene alkaloids have been reported to be produced by endophytic fungi of the genus Epichloë in perennial ryegrass (Philippe, 2016). Based on the anti-fungi activity of diterpene alkaloids (Kobayashi et al., 1998), we hypothesize that certain EFs from A. carmichaelii could produce aconitum alkaloids that inhibit these three fungal pathogens. We plan to examine the chemistry of these EFs in the future.

In this study, 171 strains of EFs were isolated and identified from six parts of $A$. carmichaelii roots, of which 29 isolates in 17 genera showed some level of anti-fungal activity. This study helps to lay a foundation for the potential association of EFs and disease resistance in A. carmichaelii.

\section{Conclusion}

Isolated from the roots of $A$. carmichaelii from six different production sites in Yunnan province, China, the root-endophytic fungi are assigned to 28 taxa. Among these identified taxa, Chaetomium and Fusarium were the dominant genera in all six 
sites while Cladosporium and Pochonia were unique dominant genera in Huize and Yulong counties. Acrostalagmus luteoalbus, Phoma sp., and Thielavia sp. displayed good anti-fungal activity against Alternaria alternate, Fusarium oxysporum f. sp. cucumerinum and Phytophthora drechsleri, providing new biological control clues for these pathogenic fungi. In subsequent experiments, further investigation on whether the endophytic fungi have the same fungal pathogen resistance in vivo was necessary. In addition, exploring the chemical composition against these fungal pathogens of endophytic fungi would be helpful for identifying the active leading chemical compounds for fungal disease control.

Acknowledgements. This work was supported by the National Natural Science Foundation of China (No. 81560622; No. 31960082) and Science and Technology Office, Yunnan Province (2016RA046).

\section{REFERENCES}

[1] Abdel-Lateff, A. (2008): Chaetominedione, a new tyrosine kinase inhibitor isolated from the algicolous marine fungus Chaetomium sp. - Tetrahedron Letters 49: 6398-6400.

[2] Aly, A. H., Edrada-Ebel, R., Wray, V., Müller, W. E., Kozytska, S., Hentschel, U., Proksch, P., Ebel, R. (2008): Bioactive metabolites from the endophytic fungus Ampelomyces sp. isolated from the medicinal plant Urospermum picroides. Phytochemistry 69: 716-1725.

[3] Bittleston, L. S., Brockmann, F., Wcislo, W., Bael, S. A. V. (2011): Endophytic fungi reduce leaf-cutting ant damage to seedlings. - Biology Letters 7: 30-32.

[4] Camargo, J. A. (1993): Must dominance increase with the number of subordinate species in competitive interactions. - Journal of Theoretical Biology 161: 537-542.

[5] Egamberdieva, D., Wirth, S., Behrendt, U., Ahmad, P., Berg, G. (2017): Antimicrobial activity of medicinal plants correlates with the proportion of antagonistic endophytes. Frontiers in Microbiology 8: 99.

[6] Fernandes, E. G., Pereira, O. L., da Silva, C. C., Bento, C. B. P., de Queiroz, M. V. (2015): Diversity of endophytic fungi in Glycine max. - Microbiological Research 181: 84-92.

[7] Fravel, D., Olivain, C., Alabouvette, C. (2003): Fusarium oxysporum and its biocontrol. New Phytologist 157: 493-502.

[8] Gao, K., Liu, X., Liu, Y., Zhu, T., Wang, S. (2002): Potential of Trichoderma harzianum and T. atroviride to control Botryosphaeria berengeriana f. sp. Piricola, the cause of apple ring rot. - Journal of Phytopathology 150: 271-276.

[9] Hashizume, Y., Fukuda, K., Sahashi, N. (2010): Effects of summer temperature on fungal endophyte assemblages in Japanese beech (Fagus crenata) leaves in pure beech stands. Botany 88: 266-274.

[10] Hunter, P. R., Gaston, M. A. (1988): Numerical index of the discriminatory ability of typing systems: an application of Simpson's index of diversity. - Journal of Clinical Microbiology 26: 2465-2466.

[11] Hyde, K. D., Soytong, K. (2008): The fungal endophyte dilemma. - Fungal Diversity 33: 163-173.

[12] Jia, M., Chen, L., Xin, H. L., Zheng, C. J., Rahman, K., Han, T., Qin, L. P. (2016): A friendly relationship between endophytic fungi and medicinal plants: a systematic review. - Frontiers in Microbiology 7: 906.

[13] Kobayashi, M., Mori, K., Kobayashi, H., Pollard, R. B., Suzuki, F. (1998): The regulation of burn-associated infections with herpes simplex virus type 1 or Candida albicans by a 
non-toxic aconitine-hydrolysate, benzoylmesaconine. Part 1: Antiviral and anti-fungal activities in thermally injured mice. - Immunology and Cell Biology 76: 202-208.

[14] Kohout, P., Těšitelová, T., Roy, M., Vohník, M., Jersáková, J. (2013): A diverse fungal community associated with Pseudorchis albida (Orchidaceae) roots. - Fungal Ecology 6: $50-64$.

[15] Kusari, S., Spiteller, M. (2012): Metabolomics of Endophytic Fungi Producing Associated Plant Secondary Metabolites: Progress, Challenges and Opportunities. - In: Prasain, J. (ed.) Metabolomics. InTech, London, pp. 241-266.

[16] Lecomte, C., Alabouvette, C., Edel-Hermann, V., Robert, F., Steinberg, C. (2016): Biological control of ornamental plant diseases caused by Fusarium oxysporum: a review. - Biological Control 101: 17-30.

[17] Leutou, A. S., Yun, K., Son, B. W. (2016): New production of antibacterial polycyclic quinazoline alkaloid, Thielaviazoline, from anthranilic acid by the Marine-Mudflatderived fungus Thielavia sp. - Natural Product Sciences 22: 216-219.

[18] Li, Y., Song, Y., Liu, J., Ma, Y., Tan, R. (2005): Anti-Helicobacter pylori substances from endophytic fungal cultures. - World Journal of Microbiology and Biotechnology 21: 553-558.

[19] Li, Z. Y., Chen, Y. W., Yang, L. Y., Li, S. L., Wu, S. H. (2009): Antipathogenic activity of endophytic fungi in Radix aconiti plant. - Natural Product Research and Development 21: 676-678.

[20] Liu, L., Li, Y., Liu, S., Zheng, Z., Chen, X., Zhang, H., Guo, L., Che, Y. (2009): Chloropestolide $\mathrm{A}$, an antitumor metabolite with an unprecedented spiroketal skeleton from Pestalotiopsis fici. - Organic Letters 11: 2836-2839.

[21] Lu, S. J., Wang, Y. Y., He, L. H. (2014): Heavy metal pollution and ecological risk assessment of paddy soils around a $\mathrm{Pb}-\mathrm{Zn}$ mine in Huize country. - Ecology and Environmental Sciences 23: 1832-1838.

[22] Marwah, R. G., Fatope, M. O., Deadman, M. L., Al-Maqbali, Y. M., Husband, J. (2007): Musanahol: a new aureonitol-related metabolite from a Chaetomium sp. - Tetrahedron 63: 8174-8180.

[23] Mishra, A., Gond, S. K., Kumar, A., Sharma, V. K., Verma, S. K., Kharwar, R. (2012): Sourcing the Fungal Endophytes: A Beneficial Transaction of Biodiversity, Bioactive natural Products, Plant Protection and Nanotechnology. - In: Satyanarayana, T. et al. (eds.) Microorganisms in Sustainable Agriculture and Biotechnology. Springer, New Delhi, pp. 581-612.

[24] Morsy, M. R., Oswald, J., He, J., Tang, Y., Roossinck, M. J. (2010): Teasing apart a three-way symbiosis: transcriptome analyses of Curvularia protuberata in response to viral infection and heat stress. - Biochemical and Biophysical Research Communications 401: 225-230.

[25] Nascimento, T., Oki, Y., Lima, D., Almeida-Cortez, J., Fernandes, G. W., Souza-Motta, C. (2015): Biodiversity of endophytic fungi in different leaf ages of Calotropis procera and their antimicrobial activity. - Fungal Ecology 14: 79-86.

[26] Newman, D. (2015): Hot topics in Pharmacognosy: belated recognition of old friends? The ASP Newsletter 51: 16-18.

[27] Nisa, H., Kamili, A. N., Nawchoo, I. A., Shafi, S., Shameem, N., Bandh, S. A. (2015): Fungal endophytes as prolific source of phytochemicals and other bioactive natural products: a review. - Microbial Pathogenesis 82: 50-59.

[28] Niu, C., Kebede, H., Auld, D. L., Woodward, J. E., Burow, G., Wright, R. J. (2010): A safe inexpensive method to isolate high quality plant and fungal DNA in an open laboratory environment. - African Journal of Biotechnology 7: 2818-2822.

[29] Philippe, G. (2016): Lolitrem B and indole diterpene alkaloids produced by endophytic fungi of the genus Epichloë and their toxic effects in livestock. - Toxins 8: 47.

[30] Qin, S., Li, J., Chen, H. H., Zhao, G. Z., Zhu, W. Y., Jiang, C. L., Xu, L. H., Li, W. J. (2009): Isolation, diversity, and antimicrobial activity of rare actinobacteria from 
medicinal plants of tropical rain forests in Xishuangbanna, China. - Applied and Environmental Microbiology 75: 6176-6186.

[31] Qiu, P., Feng, Z. X., Tian, J. W., Lei, Z. C., Wang, L., Zeng, Z. G., Chu, Y. W., Tian, Y. Q. (2015): Diversity, bioactivities, and metabolic potentials of endophytic actinomycetes isolated from traditional medicinal plants in Sichuan, China. - Chinese Journal of Natural Medicines 13: 942-953.

[32] Ratnaweera, P. B., de Silva, E. D., Williams, D. E., Andersen, R. J. (2015): Antimicrobial activities of endophytic fungi obtained from the arid zone invasive plant Opuntia dillenii and the isolation of equisetin, from endophytic Fusarium sp. - BMC Complementary and Alternative Medicine 15: 220.

[33] Rodríguez, K., Stchigel, A., Guarro, J. (2002): Three new species of Chaetomium from soil. - Mycologia 94: 116-126.

[34] Shannon, C. E., Wiener, W. (1963): The Mathematical Theory of Communication. University of Illinois Press, Urbana.

[35] Silva, H. S., Tozzi, J. P., Terrasan, C. R., Bettiol, W. (2012): Endophytic microorganisms from coffee tissues as plant growth promoters and biocontrol agents of coffee leaf rust. Biological Control 63: 62-67.

[36] Steinberg, C., Lecomte, C., Alabouvette, C., Edel-Hermann, V. (2016): Root Interactions with Nonpathogenic Fusarium Oxysporum. - In: Vos, C., Kazan, K. (eds.) Belowground Defence Strategies in Plants. Springer, Switzerland, pp. 281-299.

[37] Strobel, G., Daisy, B., Castillo, U., Harper, J. (2004): Naturalproducts from endophytic microorganisms. - Journal of Natural Products 67: 257-268.

[38] Su, Y. Y., Guo, L. D., Hyde, K. D. (2010): Response of endophytic fungi of Stipa grandis to experimental plant function group removal in Inner Mongolia steppe, China. - Fungal Diversity 43: 93-101.

[39] Sun, X., Guo, L. D., Hyde, K. (2011): Community composition of endophytic fungi in Acer truncatum and their role in decomposition. - Fungal Diversity 47: 85-95.

[40] Webber, J. (1981): A natural biological control of Dutch elm disease. - Nature 292: 449451.

[41] Xiang, L., Gong, S., Yang, L., Hao, J., Xue, M. F., Zeng, F. S., Zhang, X. J., Shi, W. Q., Wang, H., Yu, D. (2016): Biocontrol potential of endophytic fungi in medicinal plants from Wuhan Botanical Garden in China. - Biological Control 94: 47-55.

[42] Xiao, P., Wang, F., Gao, F., Yan, L., Chen, D., Liu, Y. (2006): A pharmacophylogenetic study of Aconitum L. (Ranunculaceae) from China. - Acta Phytotaxonomica Sinica 44: 146.

[43] Yuan, Z. L., Zhang, C. L., Lin, F. C., Kubicek, C. P. (2010): Identity, diversity, and molecular phylogeny of the endophytic mycobiota in the roots of rare wild rice (Oryza granulate) from a nature reserve in Yunnan, China. - Applied and Environmental Microbiology 76: 1642-1652.

[44] Zhang, S. P., Huang, R., Li, F. F., Wei, H. X., Fang, X. W., Xie, X. S., Lin, D. G., Wu, S. H., He, J. (2016): Antiviral anthraquinones and azaphilones produced by an endophytic fungus Nigrospora sp. from Aconitum carmichaeli. - Fitoterapia 112: 85-89.

[45] Zhou, G., Tang, L., Zhou, X., Wang, T., Kou, Z., Wang, Z. (2015): A review on phytochemistry and pharmacological activities of the processed lateral root of Aconitum carmichaeli Debeaux. - Journal of Ethnopharmacology 160: 173-193. 